\title{
Derivaciones urinarias laparoscópicas
}

\author{
Alonso y Gregorio S, Álvarez Maestro M, Cabrera Castillo PM, Tabernero Gómez A, \\ Cansino Alcaide R, Cisneros Ledo J, De la Peña Barthel JJ.
}

\author{
Hospital Universitario La Paz. Madrid
}

Actas Urol Esp. 2008;32(9):908-915

\section{RESUMEN}

DERIVACIONES URINARIAS LAPAROSCÓPICAS

Introducción: El tratamiento del cáncer vesical infiltrante y el carcinoma superficial de alto grado recidivante está claramente establecido en la cistectomía radical y linfadenectomía pelviana. Uno de los campos que más dudas plantea en su abordaje por vía laparoscópica es el tratamiento radical del cáncer vesical, que exige experiencia en cirugía laparoscópica pelviana y en el que la derivación urinaria presenta grandes dificultades técnicas a su abordaje laparoscópico.

Objetivos: El objetivo fundamental de este artículo es comunicar nuestros resultados con las derivaciones urinarias tras cistectomía radical laparoscópica en las que hemos llevado a cabo las anastomosis ureteroileales por vía laparoscópica.

Material y Métodos: Desde enero del 2005 hasta diciembre 2007 hemos completado 67 cistectomías radicales laparoscópicas. Hemos realizado un total de 28 derivaciones urinarias laparoscópicas según nuestra técnica, siendo en 7 pacientes enterocistoplastias de sustitución con una edad media de 54,85 años y en 21 pacientes ureteroileostomía cutánea con una edad media de 69,15 años.

Resultados: El tiempo quirúrgico medio de la enterocistoplastia con anastomosis uretral y ureteral laparoscópicas es de $5 \mathrm{~h}$ y $30 \mathrm{~min}$. Para la ureteroileostomía cutánea con anastomosis ureteral laparoscópica el tiempo quirúrgico medio ha sido de $4 \mathrm{hs}$. y $30 \mathrm{~min}$. Hemos tenido 1 caso de fuga urinaria en las neovejigas laparoscópicas y 3 casos en los conductos ileales laparoscópi$\cos (14 \%)$. No hemos tenido ningún caso de dehiscencia intestinal ni de estenosis ureteroileal. La estancia media de las neovejigas es de 13,6 días para el 85\% de los casos y de 11,8 días para el 77,7\% de las ureteroileostomías laparoscópicas.

Discusión: La cistectomía radical laparoscópica constituye un procedimiento todavía relegado a centros con gran experiencia en cirugía laparoscópica. El procedimiento más aceptado por la mayoría de los grupos incluye realizar la cistectomía por vía laparoscópica y la derivación urinaria por vía abierta. No existen evidencias sobre las posibles ventajas de las derivaciones urinarias por vía laparoscópica. En cualquier caso, son necesarios estudios comparativos para definir claramente el papel de la cirugía laparoscópica en las derivaciones urinarias.

Palabras clave: Laparoscopia. Cistectomía. Derivación urinaria. Neovejiga. Conducto ileal.

\section{ABSTRACT}

LAPAROSCOPIC URINARY DIVERSIONS

Introduction: The treatment of the invasive bladder cancer and the recurrent T1G3 is clearly established in radical cystectomy and pelvic linphadenectomy. One of the fields where the laparoscopic approach implies more doubts is the treatment of bladder cancer, because it requires experience enough in pelvic laparoscopic surgery and the urinary diversion implies great technical difficulties to the laparoscopic approach.

Objetives: The main goal of this article is to report our results with the urinary diversion after laparoscopic radical cystectomy where the ureteral anastomosis has been performed by a laparoscopic approach.

Methods: From January 2005 to December 2007 we have performed 67 laparoscopic radical cistectomies. We conducted 28 laparoscopic urinary diversions with our technique: 7 neobladder with an average age of 54,85 years and 21 ileal conduit with an average age of 69,15 years.

Results: The averagesurgical time for enterocistoplasty with laparoscopic urethral and ureteral anastomosis is of 5 hours and 30 minutes. For the cutaneous ureteroileostomy with laparoscopic ureteral anastomosis the average surgical time has been of 4 hours and 30 minutes. We have had 1 case of urinary leakage in the laparoscopic neobladder and 3 cases in the laparoscopic ileal conduit (14\%). We have not intestinal dehiscence nor ileal-ureteric stenosis. The average hospital stay for laparoscopic neobladder is for the $85 \%$ of cases of 13,6 days , and of 11,8 days for the $77,7 \%$ ofileal ureteric laparoscopies.

Discussion: The laparoscopic radical cystectomy is still a procedure reserved for groups with great experience in laparoscopic surgery. Nowadays, the most accepted procedure by most groups includes perform the cystectomy by a laparoscopic approach and the urinary diversion by an open approach. There is no evidence of the advantages of laparoscopic urinary diversion. It is necessary to perform comparative studies to clearly define the role of laparoscopic surgery in the urinary diversion.

Key words: Laparoscopy. Cystectomy. Urinary diversion. Neobladder. Ileal conduit. 
$\mathrm{E}^{\prime}$ tratamiento del cáncer vesical infiltrante y el carcinoma superficial de alto grado recidivante está claramente establecido en la cistectomía radical y linfadenectomía pelviana. Con el tratamiento quirúrgico standard por vía abierta la tasa de mortalidad es inferior al 3\% y la tasa de morbilidad postoperatoria (30 días) excede el 30\%, siendo aún superior en pacientes de edad avanzada ${ }^{1-4}$.

Desde que nuestro grupo iniciara su programa de cirugía laparoscópica en el año 2002 la progresión en este campo en nuestro medio ha sido exponencial $^{5}$. Desde entonces hemos realizado más de 1.400 procedimientos laparoscópicos de diversa índole. Uno de los campos que más dudas plantea en su abordaje por vía laparoscópica es el tratamiento radical del cáncer vesical. Por un lado exige experiencia suficiente en cirugía laparoscópica pelviana y plantea requerimientos oncológicos estrictos como puede ser la linfadenectomía, y por otro la derivación urinaria presenta grandes dificultades técnicas a su abordaje laparoscópico.

Es importante recordar que las primeras cistectomías radicales laparoscópicas fueron descritas hace más de una década ${ }^{6-8}$. Los beneficios del abordaje laparoscópico han sido definidos en una menor pérdida sanguínea y un menor dolor postoperatorio, que como consecuencia se traducen en una precoz recuperación del tránsito intestinal y de la actividad global, así como beneficios cosméticos. A pesar de dichas ventajas persiste el debate sobre si la cistectomía radical es una patología que se beneficia claramente del abordaje laparoscópico. Por otro lado, diversos grupos han desarrollado todo el procedimiento quirúrgico por vía laparoscópica en un intento de potenciar las ventajas de la cirugía laparoscópica. En el año 2000, Gill et al. describieron el primer conducto ileal puramente laparoscópico y Gupta et al. Posteriormente en el año 2002, mientras que Turk et al. en el año 2001 describen el Mainz II puramente laparoscópico y nuevamente Gill et al.. en el año 2002 hacen lo propio con el Studer laparoscópico. Existe, por tanto, una voluntad por imponer el procedimiento por vía laparoscópica también en el campo del cáncer vesical ${ }^{9-12}$.

En Enero del 2005 realizamos la primera cistectomía radical laparoscópica en nuestro centro. El objetivo fundamental de este artículo es comunicar nuestros resultados con las derivaciones urinarias tras cistectomía radical laparoscópica en las que hemos llevado a cabo las anastomosis ureteroileales por vía laparoscópica.

\section{MATERIAL Y METODOS}

\section{Pacientes}

Desde enero del 2005 hasta diciembre 2007 hemos completado 67 cistectomías radicales laparoscópicas. Hemos realizado un total de 28 derivaciones urinarias laparoscópicas según nuestra técnica, siendo en 7 pacientes enterocistoplastias de sustitución con una edad media de 54,85 años (47-66) en 3 varones y 4 mujeres, y en 21 pacientes ureteroileostomía cutánea con una edad media de 69,15 años (60-78) en 18 varones y 3 mujeres.

Hemos llevado a cabo los primeros 26 casos a través de una minilaparotomía la totalidad de la derivación urinaria con la excepción de la anastomosis neovejiga-uretra, que en el caso de la neovejiga se realizó por vía laparoscópica. Los siguientes consecutivos 28 casos se desarrolló a través de una minilaparotomía de 5-7 $\mathrm{cm}$ de longitud lo que corresponde al tiempo intestinal, y por vía laparoscópica las anastomosis ureteroileales y vesicouretrales en las neovejigas, y las anastomosis ureteroileales tipo Bricker-Wallace II o directas en los conductos ileales. Los últimos 13 casos se han llevado a cabo a través de una laparotomía suficiente para realizar toda la derivación urinaria por vía abierta.

\section{Técnica quirúrgica}

Tras finalizar la cistectomía radical y la linfadenectomía laparoscópicas, procederemos a disecar por vía laparoscópica un túnel por detrás del mesosigma para pasar el uréter izquierdo hacia el lado derecho en los conductos ileales y en las neovejigas que lo requieran. Igualmente, antes de realizar el tiempo abierto, se procede a la liberación proximal de los uréteres y del hemicolon derecho para tener la movilidad necesaria de las estructuras implicadas en el tiempo reconstructivo.

Hemos completado 7 enterocistoplastias de sustitución por vía laparoscópica realizando por vía abierta la selección del asa, la reconstrucción del tránsito intestinal y la conformación de la cara posterior de la neovejiga y la zona donde se anastomosará a la uretra. En los 5 casos en los que se ha realizado una reimplantación ureteral tipo Le-Duc laparoscópico, hemos completado sólo parcialmente la cara anterior de una neovejiga tipo Camey II 
dejando abiertos los extremos para la reimplantación ureteral. Tras cerrar la laparotomía y reconstituir el neumoperitoneo realizaremos por vía laparoscópica en primer lugar la anastomosis neovejigauretra, posteriormente la reimplantación ureteral antirreflujo y por último completaremos el cierre de la neovejiga. En los 2 casos que hemos realizado una reimplantación ureteral directa laparoscópica hemos conformado en su totalidad por vía abierta una neovejiga tipo Hautmann (Figs. 1, 2 y 5).

También hemos completado 21 ureteroileostomías por vía laparoscópica. En todos estos casos la selección del asa ileal y la reconstrucción del tránsito intestinal se han llevado a cabo a través de una minilaparotomía. Tras reconstituir el neumoperitoneo hemos realizado 16 Bricker-Wallace II y 5 reimplantaciones directas (Bricker) laparoscópicos. En todos ellos, tras finalizar las anastomosis y comprobar su estanqueidad finalizamos el procedimiento con la conformación del estoma cutáneo (Figs. 3, 4 y 5).

Todos los cateterismos ureterales se han realizado con la ayuda de la aguja-trócar de nefroscopia y de una guía teflonada por vía percutánea y bajo control laparoscópico sin complicaciones (Fig. 6).

\section{RESULTADOS}

Desde mayo del 2006 hasta septiembre del 2007 hemos realizado un total de 21 ureteroileostomías laparoscópicas, y desde octubre del 2005 hasta abril del 2007 hemos realizado un total de 7 enterocistoplastias de sustitución laparoscópicas según las técnicas descritas.

El tiempo quirúrgico medio de la cistectomía radical y linfadenectomía laparoscópicas es actualmente de 3 h 30 min. El tiempo quirúrgico medio de la enterocistoplastia con anastomosis uretral y ureteral laparoscópicas es de $5 \mathrm{~h}$ y $30 \mathrm{~min}$, lo que hace un tiempo total medio de 9 horas. Para la ureteroileostomía cutánea con anastomosis ureteral laparoscópica el tiempo quirúrgico medio ha sido de $4 \mathrm{~h}$. y $30 \mathrm{~min}$, lo que hace un tiempo quirúrgico medio total de 8 horas. Todos los
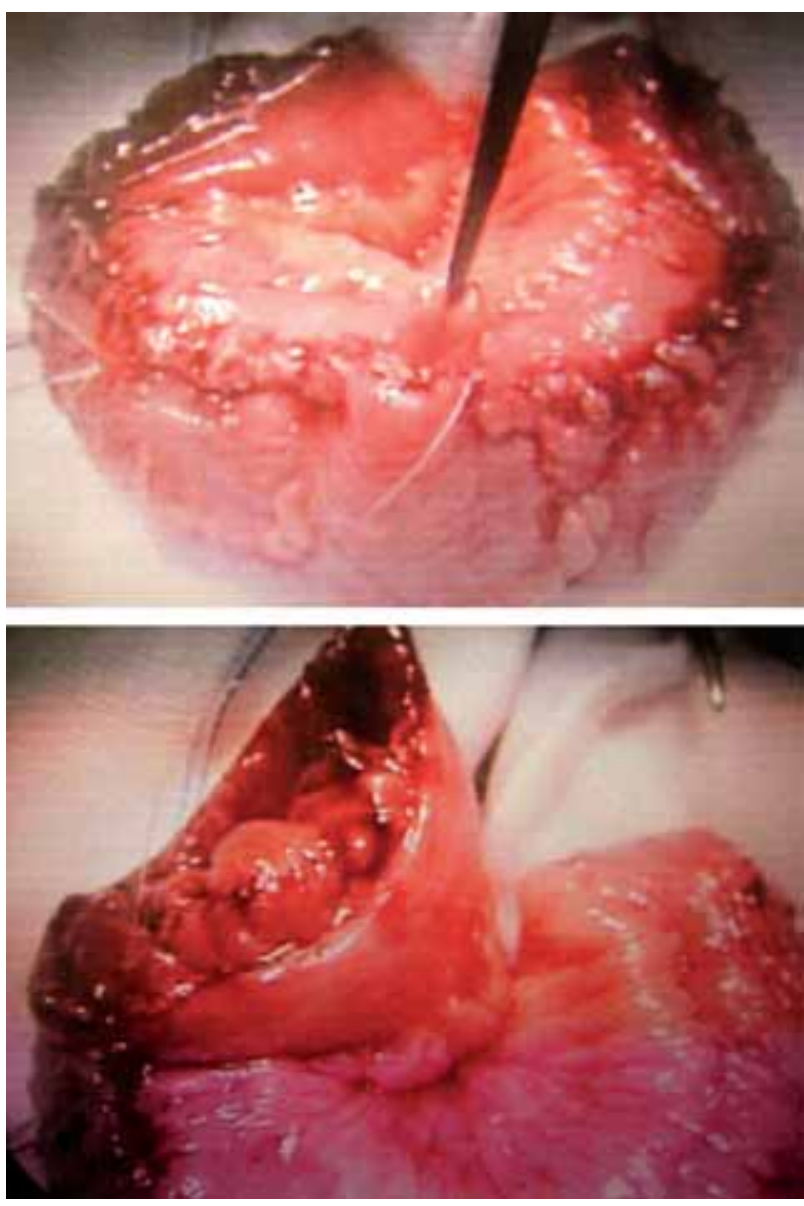

FIGURA 1. Neovejiga Camey II: extremos preparados para rimplantación ureteral tipo Le-Duc.
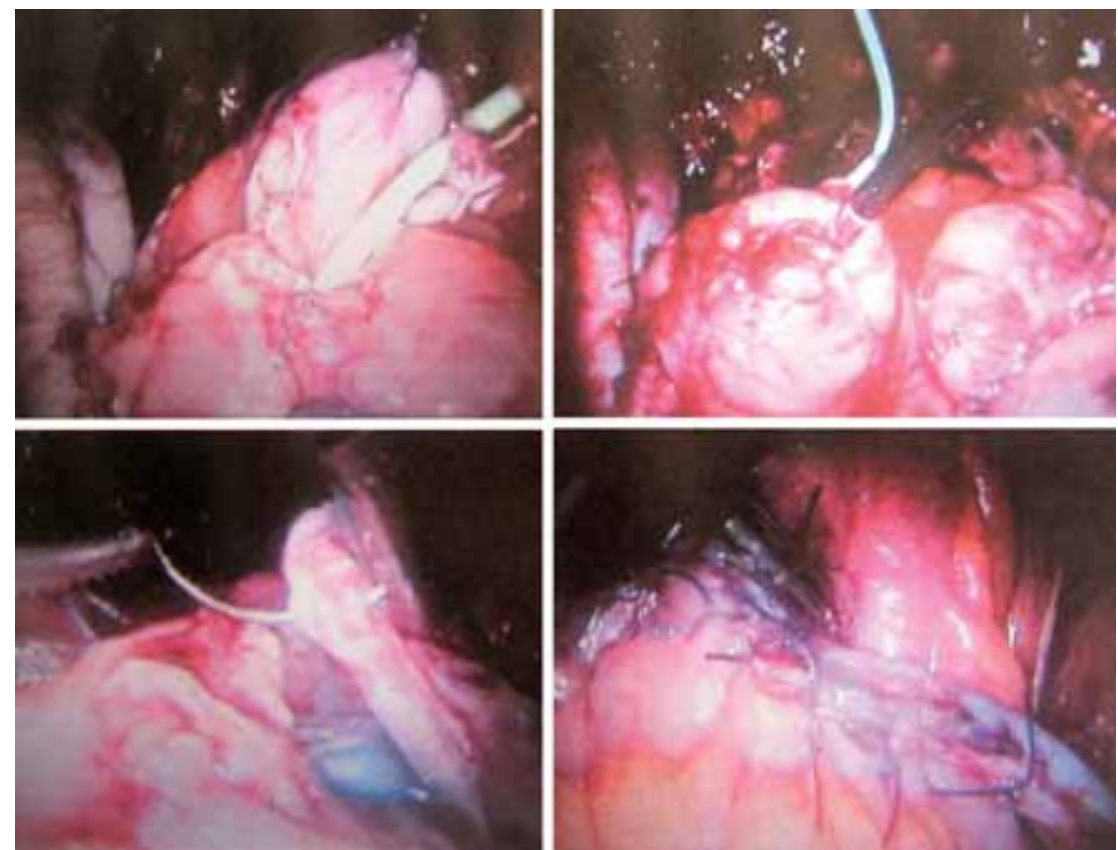

FIGURA 2. Reimplantación ureteral laparoscópica tipo Le-Duc y reimplantación ureteral directa laparoscópica en neovejiga Hautmann. 

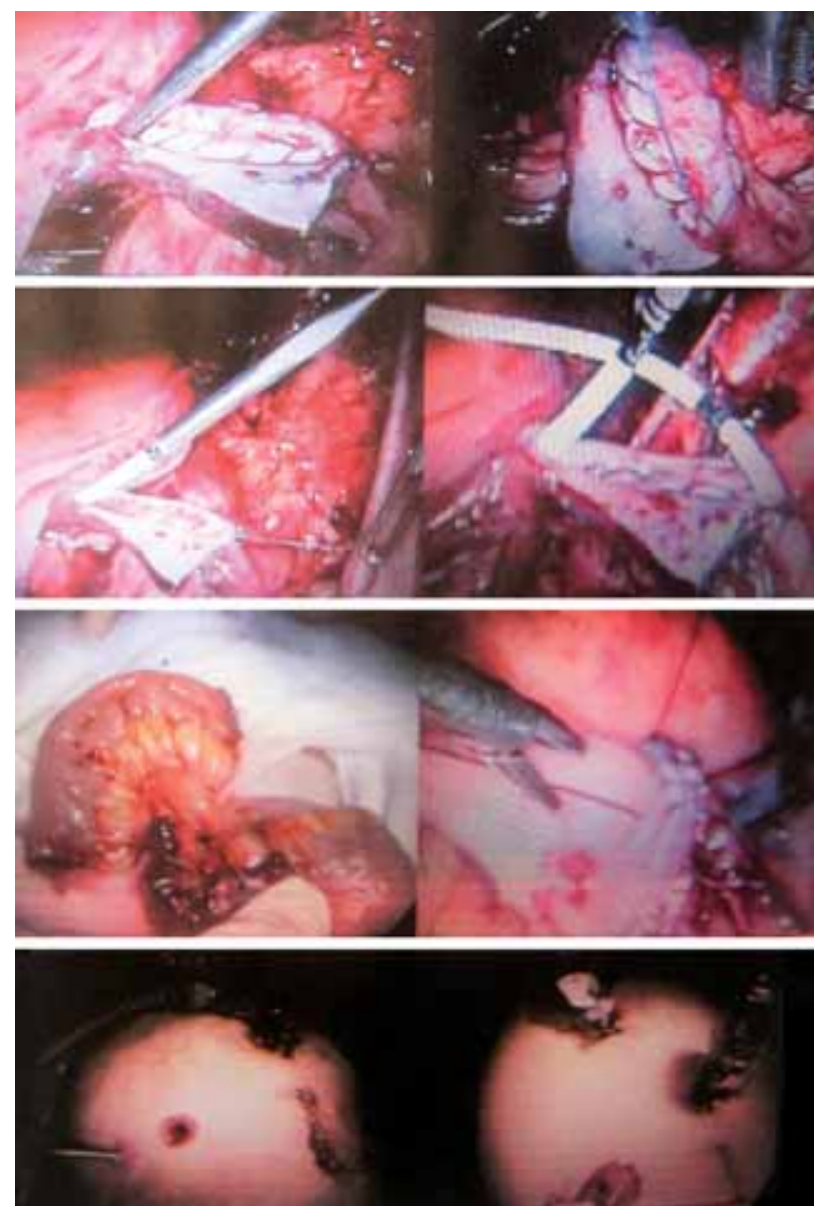

FIGURA 3. Ureteroileostomía Bricker-Wallace II laparoscópica.

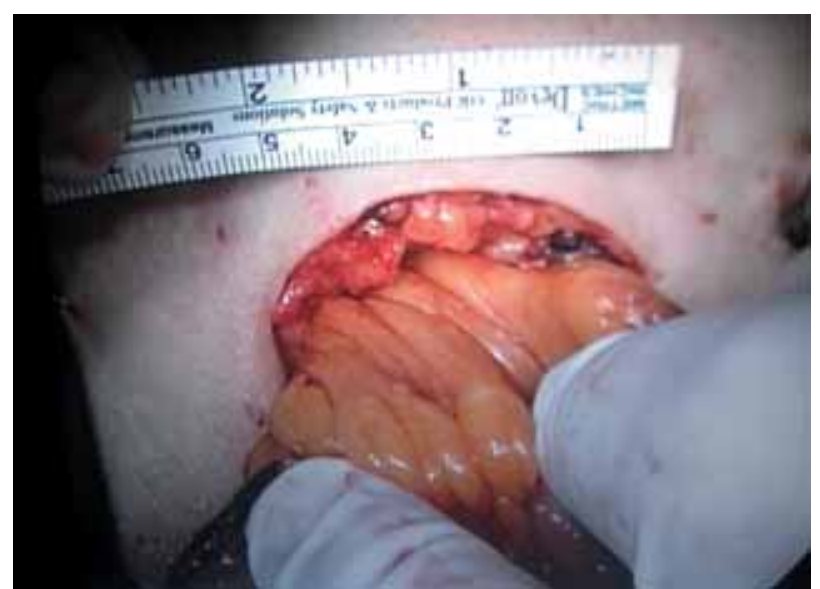

FIGURA 4. Laparatomía necesaria para el tiempo intestinal del Bricker-Wallacell.

pacientes han sido realizados por dos equipos quirúrgicos, procediendo al tiempo de exéresis un único cirujano (JCL) y del mismo modo para las derivaciones urinarias laparoscópicas un único cirujano (SAG).
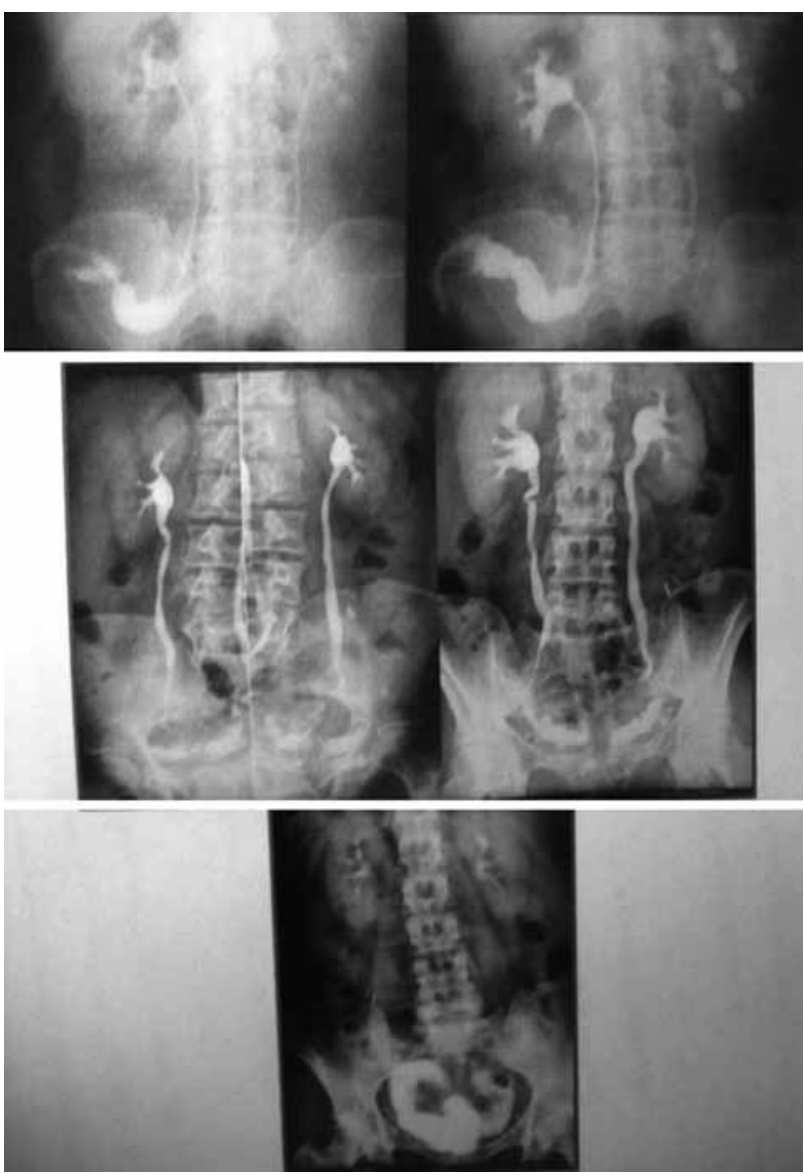

FIGURA 5. Pielografia retrógrada en Bricker-Wallace II laparoscópico y UIV en Camey II laparoscópico.

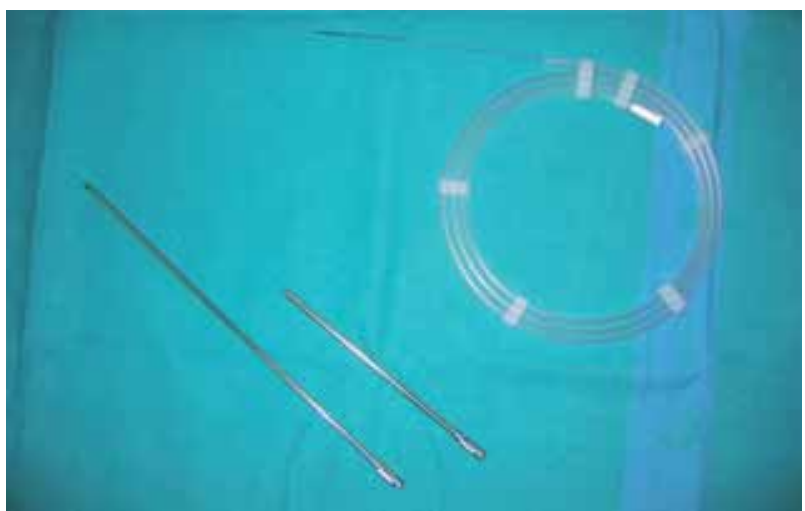

FIGURA 6. Aguja-trócar de nefroscopia.

Respecto a las complicaciones postoperatorias (30 días postoperatorios) en el grupo de las enterocistoplastias hemos tenido 1 único caso sobre 7 de fístula ureteroileal $(14,2 \%)$ resuelta mediante una nefrostomía percutánea y 1 único caso de reingreso por malfunción de la neovejiga por drenaje inadecuado de la misma, en un paciente límite por sus características anatómicas (obesidad y mesos cor- 
tos) que nos obligó a conformar una neovejiga en "Y", y que presentó un episodio de retención aguda de orina de la hemivejiga izquierda y que supuso la pérdida progresiva de la unidad renal ipsilateral durante el primer año de seguimiento.

En el grupo de las ureteroileostomías cabe destacar 3 casos de fístula ureteroileal (14,2\%) en el postoperatorio inmediato que obligó en dos de ellos a realizar sendas nefrostomías percutáneas, resolviéndose en todos los casos de forma satisfactoria. Igualmente hemos tenido 2 casos con ileo paralítico resueltos de forma conservadora y 1 caso con cuadros de suboclusión intestinal de repetición sin evidenciar en ningún estudio fuga urinaria ni trastorno digestivo.

Cabe destacar que en ningún caso de derivación urinaria laparoscópica hemos tenido que lamentar ningún caso de dehiscencia intestinal. Del mismo modo debemos resaltar que no hemos tenido ningún caso de estenosis ureteroileal en el seguimiento de estos pacientes.

La longitud de las laparotomías para el grupo de las enterocistoplastias es de 7 a $10 \mathrm{~cm}$, mientras que para los conductos ileales es de 5 a $7 \mathrm{~cm}$ (Fig. 4). En todos los casos el inicio de la deambulación se ha producido entre el $3^{\circ}$ y el $4^{\circ}$ día, mientras que el inicio de la tolerancia oral para las enterocistoplastias de sustitución se ha realizado en todos los casos entre el $3^{\circ}$ y el $6^{\circ}$ día postoperatorio, y para las ureteroileostomías entre el $3^{\circ}$ y el $5^{\circ}$ día. La estancia media para las neovejigas ha sido de 17 días, que se reduce a 13,6 (85\% de los casos) días si excluimos el paciente con fístula urinaria postoperatoria. Para los conductos ileales la estancia media sobre 18 casos es de 17,1 días, tras excluir 1 falle- cimiento postoperatorio por causas cardiovasculares, 1 paciente al que se le diagnostica en el postoperatorio una neoplasia de colon y 1 paciente por reintervención a las 24 hs. por sangrado. Si excluimos los 3 pacientes con fístula urinaria postoperatoria y 1 paciente con cuadros de suboclusión de repetición probablemente por bajo gasto esplácnico, obtenemos una estancia media de 11,8 días para el $77,77 \%$ de los casos (14 casos de un total de 18). En el grupo de los conductos ileales laparoscópicos un paciente falleció en el inmediato postoperatorio por causas cardiovasculares y 3 han fallecido por progresión de su enfermedad, mientras que en el grupo de las neovejigas laparoscópicas no ha habidos muertes postoperatorias $\mathrm{y}$ se han presentado 2 fallecimientos por progresión de la enfermedad.

Tras un seguimiento medio de 18,5 meses (10-24 meses) sobre 17 ureteroileostomías laparoscópicas (excluidos 4 fallecimientos), 15 pacientes presentan cretininas estables <1,5 mg/dl (0,69-1,5 mg/dl), 1 paciente presenta un cretinina de $2,9 \mathrm{mg} / \mathrm{dl}$. similar a la preoperatoria y un paciente presenta una cretinina estable de $2 \mathrm{mg} / \mathrm{dl}$.

Para las enterocistoplastias de sustitución, el seguimiento medio es de 22 meses (14-32 meses) sobre 6 casos (excluido 1 fallecimiento a los 6 meses del seguimiento), presentando 4 casos cretininas estables < 1,2 mg/dl (0,69-1,2 mg/dl) y 2 casos creatininas estables de 1,8 mg/dl, siendo ambos pacientes monorrenos (Tabla 1).

\section{DISCUSIÓN}

La mejor forma de abordar el tratamiento por vía laparoscópica del cáncer vesical no está definido.

Tabla 1. Resultados a corto y medio plazo de las derivaciones urinarias laparoscópicas

\begin{tabular}{|c|c|c|}
\hline & $\begin{array}{l}\text { Ureteroileostomía cutánea } \\
\text { laparoscópica }\end{array}$ & $\begin{array}{l}\text { Enterocistoplastia de sustitución } \\
\text { laparoscópica }\end{array}$ \\
\hline Numeros de casos & 21 & 7 \\
\hline Seguimiento medio & 18,5 meses $(10-24 \mathrm{~m})$ & 22 meses (14-32 m) \\
\hline Tiempo qco. Medio & 4 h 30 min (total: 8 h) & 5 h 30 min. (total: 9 h) \\
\hline Long. Laparotomia & $5-7 \mathrm{~cm}$ & $7-10 \mathrm{~cm}$ \\
\hline Fistula urinaria & $3(14,2 \%)$ & $1(14,2 \%)$ \\
\hline Tasa de reingreso & $0 \%$ & $1(14,2 \%)$ \\
\hline Dehiscencia intestinal & $0 \%$ & $0 \%$ \\
\hline Estenosis ueteroileal & $0 \%$ & $0 \%$ \\
\hline Estancia media & 11,8 dias $(17,1 \mathrm{~d})$ & 13,6 días(17 d) \\
\hline
\end{tabular}


Las ventajas de la cistectomía radical laparoscópica pueden ser discutidas por el hecho de necesitar una considerable experiencia en cirugía laparoscópica y porque los defensores del abordaje convencional disponen de los mismos medios de hemostasia que aplicamos en cirugía laparoscópica y que han mejorado los resultados en términos de sangrado. Algunos grupos han intentado potenciar los beneficios de la laparoscopia en este procedimiento realizando la derivación urinaria por vía laparoscópica9-13. Hoy en día todavía hay grupos en esta dirección e incluso los grupos con experiencia en cirugía robótica también comunican procedimientos enteramente laparoscópicos ${ }^{14-16}$.

Nuestro grupo ha realizado una clara apuesta por los procedimientos laparoscópicos desarrollando actualmente la casi totalidad de las prostatectomías, la mayoría de las cirugías retroperitoneales y de las cistectomías, así como procedimientos como el linfocele o la reimplantación ureteral por vía laparoscópica.

En Enero del 2005 iniciamos nuestro programa de cistectomías laparoscópicas. Tras los primeros 26 casos en los que realizábamos todas las derivaciones urinarias por vía abierta a través de una minilaparotomía, obtuvimos una tasa de estenosis ureteroinestinales excesivamente elevada ${ }^{17}$. Por este motivo, decidimos realizar las derivaciones por vía laparoscópica. Basándonos en la literatura de los escasos grupos con cierta experiencia en este campo desestimamos la derivación urinaria enteramente laparoscópica tanto por el inasumible tiempo quirúrgico como por la elevada tasa de complicaciones mayores ${ }^{18}$. De este modo decidimos realizar por vía abierta el tiempo intestinal y las reimplantaciones ureterales por vía laparoscópica.

Dado el evidente coste en tiempo quirúrgico que supone realizar una gran parte del procedimiento por vía laparoscópica decimos realizar dos equipos quirúrgicos que ofrece ventajas ya descritas por otros grupos ${ }^{18}$. En el caso de realizar un conducto ileal todas las laparotomías necesarias han sido de 5-7 cm de longitud, mientras que en el caso de las enterocistoplastias de sustitución la laparotomía necesaria es de entre 7 y $10 \mathrm{~cm}$ en función de la obesidad del paciente y de las características del meso ileal. Una de las ventajas evidentes de la cirugía laparoscópica en general y de la cistectomía en particular es el hecho de realizar incisiones de pequeño tamaño y obviar el empleo de retractores lo que se traduce en unos menores requerimientos analgésicos en el postoperatorio. Igualmente ofrece ventajas puesto que limita el tiempo y la longitud del paquete intestinal que se expone a la temperatura ambiente y a la manipulación por el cirujano. Tanto el menor dolor postoperatorio como la menor exposición intestinal tienen efectos beneficiosos en la recuperación del tránsito intestinal de forma más precoz ${ }^{17}$. Nosotros no nos hemos planteado en ningún caso la derivación urinaria íntegramente por vía laparoscópica puesto que obliga a unos tiempos quirúrgicos inaceptables, y por la alta tasa de complicaciones descritas por otros grupos como recientemente publicaron Haber y Gill con una tasa de reintervenciones por fístula intestinal o urinaria del $29 \%^{18}$. Dada la experiencia de la Cleveland Clinic confirmamos nuestra acertada postura al evitar realizar la continuidad intestinal por vía laparoscópica, a pesar de tener alguna experiencia positiva en enterocistoplastias de ampliación laparoscópicas ${ }^{19}$. Con nuestra técnica aseguramos la continuidad intestinal con las mismas garantías que en la cirugía convencional a través de una laparotomía mínima. Según nuestra experiencia realizar la derivación urinaria por vía abierta a través de una minilaparotomía obliga a emplear una mayor longitud ureteral cuya vascularización puede estar comprometida traduciéndose en una tasa elevada de estenosis ureterointestinales del $17 \%{ }^{17}$. En nuestra serie de derivación urinaria a través de una minilaparotomía con reimplantación ureteral laparoscópica, hemos tenido 3 fístulas urinarias en Bricker-Wallace II y en una enterocistoplastia de sustitución que han sido manejadas en todos los casos de forma conservadora con éxito. Todos estos casos pueden atribuirse a la curva de aprendizaje por tratarse en la mayoría de casos iniciales o pacientes obesos (neovejiga). En cualquier caso no tenemos ningún paciente con estenosis ureterointestinal a diferencia del grupo inicial realizado por vía abierta a través de una minilaparotomía, ni ningún caso de fístula intestinal en el postoperatorio.

Los tiempos quirúrgicos de todas las series de cistectomías radicales laparoscópicas son prolongados (6-10 h). Los tiempos quirúrgicos vienen condicionados inicialmente por la curva de aprendizaje en el tiempo de la exéresis. Muchos grupos realizan la linfadenectomía a través de la incisión por la que 
realizan la derivación urinaria. En los primeros 30 casos el tiempo de cistectomía y linfadenectomía oscilaba entre 3 y 5 horas, siendo actualmente el tiempo medio de $3 \mathrm{~h}$ y 30 min con un número de ganglios extraídos similar a las series de abierta. En los últimos 12 casos de ureterolileostomía cutánea el tiempo quirúrgico medio en el grupo laparoscópico es de 4 h 30 min mientras que en las enterocistoplastias de sustitución es de 5 h $30 \mathrm{~min}$. Por tanto, el tiempo quirúrgico medio de cistectomía radical con derivación laparoscópica es de $8 \mathrm{~h}$ para el Bricker y de $9 \mathrm{~h}$ para la neovejiga. En el caso concreto del Bricker-Wallace el tiempo laparoscópico es de de $2 \mathrm{~h}$ de media, puesto que la continuidad intestinal y la confección del estoma se realiza sin neumoperitoneo, lo que hace un total de 5 h $30 \mathrm{~min}$ de media de tiempo laparoscópico para todo el procedimiento, lo que es importante desde el punto de vista cardiovascular-anestésico.

En el grupo de las neovejigas, realizamos inicialmente las reimplantaciones ureterales tipo Le-Duc por tratarse de la técnica más frecuentemente empleada por nuestro grupo en la experiencia de cirugía abierta. Dada la tasa descrita de estenosis del $2-4 \%^{20}$ y para reducir el tiempo quirúrgico, hemos realizado 2 casos con reimplantación ureteral directa. Hemos demostrado con nuestra serie que dicho procedimiento por vía laparoscópica es viable y presenta unos resultados similares a los del grupo histórico de cistectomías abiertas. Hemos tenido tan sólo un caso de fístula urinaria resuelta mediante una nefrostomía percutánea y ninguna estenosis ureterointestinal. En el grupo de neovejiga abierta tras cistectomía radical laparoscópica los resultados son absolutamente comparables en términos de fuga urinaria y estancia postoperatoria. Cabe resaltar que no tenemos ningún caso de estenosis ureterointestinal en ninguno de los dos grupos.

Respecto a la ureteroileostomía cutánea, hemos realizado 16 Bricker-Wallace II laparoscópicos por tratarse de la técnica más frecuente en nuestro grupo, y 5 reimplantaciones directas (Bricker) laparoscópicos dado el menor tiempo quirúrgico y las tasas similares de complicaciones publicadas ${ }^{21,22}$. Los principales problemas de estenosis ureterointestinales se han presentado en este grupo de pacientes cuando se han realizado por vía abierta a través de una minilaparotomía ${ }^{17}$. A diferencia del abordaje abierto a través de una minilaparotomía, en el grupo de derivaciones laparoscópicas no tenemos ningún caso de estenosis ureteroileal. El comportamiento postoperatorio en términos de recuperación precoz del tránsito intestinal y de los requerimientos analgésicos han sido muy superiores a nuestra serie histórica de cirugía abierta y discretamente superior al grupo laparoscópico con minilaparotomía, por lo que desde de Noviembre del 2007 estamos realizando todas nuestras derivaciones por vía abierta a través de una laparotomía suficiente.

\section{CONCLUSIONES}

La cistectomía radical laparoscópica constituye un procedimiento todavía relegado a centros con gran experiencia en cirugía laparoscópica. Aunque son varios los grupos que han realizado las derivaciones íntegramente por vía laparoscópica, este abordaje se ha abandonado por el prolongado tiempo quirúrgico y la elevada tasa de complicaciones.

Actualmente, el procedimiento más aceptado por la mayoría de los grupos incluye realizar la cistectomía por vía laparoscópica y la derivación urinaria por vía abierta. En nuestra experiencia la cistectomía radical por vía laparoscópica ofrece ventajas en términos de sangrado, dolor postoperatorio, recuperación precoz del tránsito intestinal y de la actividad cotidiana, e incluso mejoras cosméticas.

Desde nuestra experiencia la enterocistoplastia de sustitución con reimplantación ureteral laparoscópica no ofrece ventajas con respecto a la reimplantación abierta, y sin embargo supone un tiempo quirúrgico más prolongado, por lo que abogamos por hacerlo a través de una laparotomía económica.

La ureteroileostomía laparoscópica es perfectamente viable hacerlo según la técnica que hemos descrito. Ofrece unos resultados postoperatorios superiores a nuestra serie histórica en términos de estancia postoperatoria y a medio plazo superiores al procedimiento a través de una minilaparotomía en tales términos. Probablemente los resultados sean similares si se realiza el procedimiento abierto a través de una laparotomía suficiente para garantizar el resultado de nuestras anastomosis. Aunque pueda haber una pequeña ventaja en términos de estancia en la reimplantación laparoscópica es poco probable que compense el tiempo quirúrgico asociado, por lo que actualmente la realizamos por vía abierta. 
En cualquier caso, son necesarios estudios comparativos para definir claramente el papel de la cirugía laparoscópica en las derivaciones urinarias.

\section{REFERENCIAS}

1. Richard E. Hautmann, Hassan Abol-Enein, Urs E. Studer et al. For the WHO, Consensus Conference on Bladder Cancer Urinary diversion. Urology 2007; 69 (suppl. 1A): 17-49.

2. Quek ML, Stein JP, Daneshmand S, Miranda G, Thangathurai D, Roffey P et al. A critical analysis of perioperative mortality from radical cystectomy. J Urol. 2006;175(3 Pt 1):886-890.

3. Maffezzini M, Gerbi G, Campodonico F, Parodi D. Multimodal perioperative plan for radical cystectomy and intestinal urinary diversion. Effect on recovery of intestinal function and occurrence of complications. Urology. 2007;69(6):1107-1111.

4. John P. Stein and Donald G. Skinner. Radical cystectomy for invasive bladder ancer: long term results of a standard procedure. World J Urol. 2006.

5. Martínez-Piñeiro, Felipe Cáceres, Carlos Sánchez et al. Learning curve of laparoscopic radical prostatectomy in a university teaching hospital: experience after the first 600 cases. Eur Urol Suppl. 2006;5:914-924.

6. Parra RO, Andrus CH, Jones JP, Bouller JD. Laparoscopic cystectomy: initial report on a new treatment for the retained bladder. J Urol. 1992;148(4):1140-1144.

7. Puppo P, Perachino M, Ricciotti G, Bozzo W, Gallucci M, Carmignani G. Laparoscopically assisted transveginal radical cystectomy. Eur Urol. 1995;27(1):80-84.

8. Sánchez de Badajoz E, Gallego Perales JL, Reche Rosado A, Gutiérrez de la Cruz JM, Jiménez Garrido A. Radical cystectomy and laparoscopic ileal conduit. Arch Esp Urol. 1993;46 (7):621-624

9. Gill IS, Fergany A, Klein EA, Kaouk JH, Sung GT, Meraney AM. Laparoscopic radical cystoprostatectomy with ileal conduit performed completely intracorporeally: the initial 2 cases. Urology 2000;56(1):26-29.

10. Gupta NP, Gill IS, Fergany A. Laparoscopic radical cystectomy with intracorporeal ileal conduit diversion: five cases with a 2year follow-up. BJU Int. 2002;90(4):391-396

11. Türk I, Deger S, Winkelmann B, Schönberger B, Loening SA. Laparoscopic radical cystectomy with continent urinary diversion (rectal sigmoid pouch) performed completely intracorporeally: the initial five cases. J Urol. 2001;165(6 Pt 1): 1863-1866.
12. Gill IS, Kaouk JH, Meraney AM, Desai MM, Ulchaker JC, Klein EA et al. Laparoscopic radical cystectomy and continent orthotopic ileal neobladder performed completely intracorporeally: the initial experience. J Urol. 2002;168(1):13-18.

13. Potter SR, Charambura TC, Adams JB and Kavoussi LR. Laparoscopic ileal conduit: five years follow-up. Urology. 2000;56(1): 22-25.

14. Sala LG, Matsunaga GS, Corica FA, Ornstein DK. Robot-assisted laparoscopic radical custoprostatectomy and totally intracorporeal ileal neobladder. J Endourol. 2006;20(4):233-235.

15. Beecken WD, Wolfram M, Engl T, Bentas W, Probst M, Blaheta $\mathrm{R}$, et al. Robotic-assisted laparoscopic radical cystectomy and intra-abdominal formation of an orthotopic ileal neobladder. Eur Urol. 2003;44(3):337-339.

16. Balaji KC, Yohannes P, McBride CL, Oleynikov D, Hemstreet GP 3rd. Feasibility of robot-assisted totally intracorporeal laparoscopic ileal conduit urinary diversion: initial results of a single institutional pilot study. Urology. 2004;63(1):51-55.

17. JR Cansino, Jesus Cisneros, Sergio Alonso et al. Laparoscopic radical cystectomy: initial series and analysis of results. Eur Urol Suppl. 2006; 5:956-961.

18. Georges-Pascal Haber, Steven C Campbell, Amr F Fergany, Gill IS et al. Perioperative outcomes with laparoscopic radical cystectomy: Pure laparoscopic and open-assisted laparoscopic approaches. Urology. 2007;70:910-915.

19. Nuñez Mora C, Cansino Alcaide R, Alonso Gregorio S et al. Enterocistoplastia de ampliación laparoscópica: experiencia inicial. Actas Urol Esp. 2007;37(1):17-22.

20. De Carli P, Micali S, O'Sullivan D, Mainiero G, Cusumano G, Fattahi H. Ureteral anastomosis in the orthotopic ileal neobladder: comparision of 2 techniques. J Urol. 1997;157(2): 469-471.

21. Evangelidis A, Lee EK, Karellas ME, Thrasher JB, Holzbeierlein JM. Evaluation of ureterointestinal anastomosis: Wallace vs Bricker J Urol. 2006; 175(5):1755-1758.

22. Kouba E, Sands M, Lentz A, Wallen E, Pruthi RS. A comparision of the Bricker versus Wallace ureteroileal anastomosis in patients undergoing urinary diversion for bladder cancer. $\mathrm{J}$ Urol. 2007;179(3 Pt 1):945-948.

Correspondencia autor: Dr. S. Alonso y Gregorio

Servicio de Urología. Hospital Universitario La Paz

Paseo de la Castellana, 261 - 28046 Madrid. Tel.: 917277305

E-mail autor: garavelos@terra.es

Información artículo: Original - Laparoscopia

Trabajo recibido: junio 2008

Trabajo aceptado: julio 2008 